\title{
Distinguishing causality principles
}

\author{
Miklós Rédei \\ Department of Philosophy, Logic and Scientific Method \\ London School of Economics and Political Science \\ Houghton Street, London WC2A 2AE, England \\ M.Redei@lse.ac.uk \\ Iñaki San Pedro \\ Department of Logic and Philosophy of Science \\ Complutense University Madrid \\ Ciudad Universitaria, 28040 Madrid, Spain \\ inaki.sanpedro@filos.ucm.es
}

\begin{abstract}
We distinguish two sub-types of each of the two causality principles formulated in connection with the Common Cause Principle in Hen05. and raise and investigate the problem of logical relations among the resulting four causality principles. Based in part on the analysis of the status of these four principles in algebraic quantum field theory we will argue that the four causal principles are non-equivalent.
\end{abstract}

\section{Introduction}

Distinguishing and comparing carefully causality principles is important when it comes to the question of whether a fundamental physical theory is compatible with "causality". Motivated to a large extent by the well-known difficulties that stand in the way of explaining quantum (EPR) correlations causally, Henson [Hen05] proposed two apparently different causality principles (SO1 and SO2 in the notation of [Hen05]) and proved that the two principles are in fact equivalent. The equivalence claim states, roughly, that two requirements about the localizability of a common cause of a correlation between causally disjoint events $A$ and $B$ are equivalent: localizability within the mutual causal past of $A$ and $B$ (this is SO1) and localizability within the joint causal past of $A$ and $B$ (this is SO2). The aim of this paper is to distinguish further two sub-types of both SO1 and SO2 and to raise and investigate the problem of logical relations among the resulting four causality principles.

The need to distinguish the further types arises from the fact that the $\mathrm{SO} 1 \Leftrightarrow \mathrm{SO} 2$ equivalence seems counterintuitive: the mutual past being part 


\section{Introduction}

of the joint past, common causes localized in the mutual past are localized in the joint past as well, hence $\mathrm{SO} 1 \Rightarrow \mathrm{SO} 2$ corresponds to our intuition; however, the converse implication $\mathrm{SO} 1 \Leftarrow \mathrm{SO} 2$ does not. The intuition about this asymmetry is supported by specific results about existence of localized common causes in relativistic quantum field theory: The equivalence SO1 $\Leftrightarrow \mathrm{SO} 2$ indicates that algebraic relativistic quantum field theory, which predicts correlations between observables localized in causally disjoint regions, would satisfy the Common Cause Principle in the sense of providing causal explanations of spacelike correlations in terms of common causes localized in the mutual causal past of the spacelike separated spacetime regions containing the correlated observables because common causes localized in the joint pasts have been shown to exist [RS02, [RS07. Given that no proof of stricter localizability of common causes in algebraic quantum field theory could be given so far, and in view of the fact that recent further investigations of the status of the common cause principle in lattice quantum field theory seem to indicate that the two localizability conditions are not equivalent [HSV12, a careful scrutiny of the equivalence claim and of its proof is called for.

A look at the proof of the equivalence claim reveals that the crucial feature of $\mathrm{SO} 2$ that the proof of the implication $\mathrm{SO} 2 \Rightarrow \mathrm{SO} 1$ is based on is that $\mathrm{SO} 2$ allows to consider correlations between events associated with "causally infinite" regions and requires such correlations to have common causes localized in the joint past of the causally infinite regions. We will argue that this makes SO2 extremely strong and that it is both physically and intuitively justified to distinguish such "infinite SO2" from a weaker, "finite SO2" principle that restricts the correlations for which common causes are required to exist to correlations between events in "causally finite" regions (see Definition 3 for causally infinite and causally finite regions). A similar distinction can be made in connection with $\mathrm{SO} 1$, and thus one has four distinct causality principles: finite and infinite $\mathrm{SO} 2$, on one hand, and finite and infinite $\mathrm{SO} 1$ on the other. The question arises then what the relation of these four principles are. This seems to be a difficult problem that remains largely open. Henson proves the equivalence of infinite SO2 and infinite SO1 - although we detect what appears a slight gap in the proof of the implication [infinite SO1 $\Rightarrow$ infinite SO2], see the end of Section 5. This gap questions whether the implication [finite $\mathrm{SO} 1 \Rightarrow$ finite $\mathrm{SO} 2$ ] holds, but we do not have a counterexample to the implication. It will be shown however that Henson's method does not prove the implication [ finite $\mathrm{SO} 2 \Rightarrow$ finite $\mathrm{SO} 1$ ], and that it does not prove the implication [finite $\mathrm{SO} 2 \Rightarrow$ infinite $\mathrm{SO} 1$ ] either, and we will argue, although we are unable to provide a strict formal proof, that the finite SO2 does not entail even the finite SO1.

Henson's formulates the causality principles SO1 and SO2 within a very elaborate formal framework that links explicitly probabilistic concepts to spatiotemporal notions. Section 2 recalls briefly this framework and some 
definitions needed to formulate the two causality principles SO1 and SO2, which will be stated explicitly in Section 3 together with Henson's proof of their equivalence. Section 4 analyzes the equivalence proof and motivates the distinction between finite and infinite versions of SO1 and SO2. Finally, Section 5 discusses the problem of relation of the principles, arguing that they are not equivalent.

\section{Events, probabilities and least domain of decid- ability}

Henson creates a formal framework in order to relate probabilistic concepts to spatiotemporal notions, the latter understood as including concepts of non-probabilistic causality. Here we recall this framework following the notations of [Hen05] closely.

Let $\mathbb{S}$ be some spacetime structure. Henson leaves the nature of $\mathbb{S}$ unspecified except that he assumes a causal (partial) order $<$ defined on $\mathbb{S}$ that expresses causal precedence between elements $x, y$ in $\mathbb{S}$ : if $x<y$ then $x$ is in the causal past of $y \underline{1}$ Given $(\mathbb{S},<)$ the past $J^{-}(x)$ of $x \in \mathscr{X}$, is defined as the set of all points that precedes $x$ in the causal order, plus $x$ itself, i.e.

$$
J^{-}(x)=\left\{x_{p} \in \mathbb{S}: x_{p}<x\right\} \cup\{x\}
$$

The past of $\mathscr{X} \subset \mathbb{S}$ is then defined [Hen05, p. 523] as the union of the pasts of all points in the region:

$$
J^{-}(\mathscr{X})=\bigcup_{x \in \mathscr{X}} J^{-}(x)
$$

Given two regions $\mathscr{X}$ and $\mathscr{Y}$ in $(\mathbb{S},<)$ one can define the following regions:

Mutual past: $\mathscr{P}_{1}(\mathscr{X}, \mathscr{Y})=J^{-}(\mathscr{X}) \cap J^{-}(\mathscr{Y})$

Joint past: $J^{-}(\mathscr{X}) \cup J^{-}(\mathscr{Y})$

Truncated joint past: $\mathscr{P}_{2}(\mathscr{X}, \mathscr{Y})=\left(J^{-}(\mathscr{X}) \cup J^{-}(\mathscr{Y})\right) \backslash(\mathscr{X} \cup \mathscr{Y})$

\footnotetext{
${ }^{1}$ Henson lists as examples of such causally ordered sets weakly causal Lorentzian manifolds and causal sets [Hen05, p. 523]. Weakly causal Lorentzian manifolds are manifolds that contain no causally closed curves; an example is the Minkowski spacetime. If the causal partial order $<$ defined in $\mathbb{S}$ is transitive, acyclic and locally finite, then $\mathbb{S}$ is called a causal set. Causal sets so defined then constitute the discreet equivalent to spacetime manifolds equipped with causal structure. (Discreetness is introduced by the locally finite property of the order relation in a causal set, i.e. $\forall x, z \in \mathbb{S}$, $\operatorname{card}\{y \in \mathbb{S}: x<y<z\}<\infty$. See Sor03 for details.)
} 
Note that the terminology in Hen05 is slightly different: Henson calls the truncated joint past $\mathscr{P}_{2}(\mathscr{X}, \mathscr{Y})$ "joint past" and does not define joint past explicitly. We will use the terminology introduced above.

Two regions $\mathscr{X}$ and $\mathscr{Y}$ are defined to be space-like separated if

$$
\begin{aligned}
& J^{-}(\mathscr{X}) \cap \mathscr{Y}=\emptyset, \\
& \mathscr{X} \cap J^{-}(\mathscr{Y})=\emptyset .
\end{aligned}
$$

In the notations of [Hen05], a classical probability measure space is a triplet $(\Omega, \Sigma, \mu)$ where $\Omega$ is "the space of all possible histories of the system" [Hen05, p. 521] to be described probabilistically, $\Sigma$ is an "appropriate $\sigma$ algebra of subsets of $\Omega$ " [Hen05, p. 521] and $\mu: \Sigma \rightarrow \mathbb{R}$ is a probability measure "obeying the usual axioms" [Hen05, p. 521]. The link between $(\Omega, \Sigma, \mu)$ and spatiotemporal concepts is given by a map

$$
\text { dom }: \Sigma \rightarrow \operatorname{pow}(\mathbb{S})
$$

where pow $(\mathbb{S})$ denotes the power set of $\mathbb{S}$. The region $\operatorname{dom}(A)$ is called the least domain of decidability of event $A \in \Sigma$ - intuitively, $\operatorname{dom}(A)$ is the unique spacetime region such that if we know all the properties of the history in it we can decide, without any further information, whether $A$ has occurred or not.

The properties of dom are fixed axiomatically by formulating requirements that it should satisfy. Following [Hen05, p. 524] we list the axioms below.

\section{Properties of dom:}

For all countable subsets $\Lambda \subset \Sigma$ we have

(i) If $\operatorname{dom}(X) \cap \operatorname{dom}(Y)=\emptyset \quad \forall X, Y \in \Lambda$, such that $X \neq Y$ then

$$
\operatorname{dom}\left(\bigcap_{X \in \Lambda} X\right)=\bigsqcup_{X \in \Lambda} \operatorname{dom}(X)
$$

where $\bigsqcup$ stands for the 'disjoint union'.

(ii) If $\operatorname{dom}(X)=\operatorname{dom}(Y) \quad \forall X, Y \in \Lambda$ then

$$
\operatorname{dom}\left(\bigcap_{X \in \Lambda} X\right) \subset \operatorname{dom}(Y), \quad \forall Y \in \Lambda .
$$

(iii) $\operatorname{dom}\left(X^{c}\right)=\operatorname{dom}(X), \quad \forall X \in \Sigma$.

(iv) $\forall Z \in \Sigma$ such that $\operatorname{dom}(Z)=\mathscr{X} \sqcup \mathscr{Y}, Z$ is a member of the $\sigma$-algebra generated by $\Gamma(\mathscr{X}) \cup \Gamma(\mathscr{Y})$, where $\Gamma(\mathscr{X})=\{X \in \Sigma: \operatorname{dom}(X) \subset \mathscr{X}\}$.

A further notion that will be important in the discussion of the common cause principle is the full specification of a region: 
Definition 1. Event $F \in \Sigma$ is a full specification of a region $\mathscr{R}$ if $\operatorname{dom}(F) \subset$ $\mathscr{R}$, and for all $X \in \Sigma$ it holds that, if $\operatorname{dom}(X) \subset \mathscr{R}$, then either $F \subset X$ or $F \subset X^{c}$.

$\Phi(\mathscr{R})$ denotes the set of full specifications of region $\mathscr{R}$. Henson proves the following proposition:

Proposition 1 (Lemma 3 in [Hen05]). The full specification of any region is a partition of $\Omega$.

\section{Two Common Cause Principles: SO1 and SO2 and the Equivalence Claim}

The next definition of common cause was given by Reichenbach [Rei56] and is standard in the literature.

Definition 2. Assume that $A, B \in \Sigma$ are positively correlated in $\mu$ :

$$
\mu(A \cap B)>\mu(A) \mu(B)
$$

Event $C \in \Sigma$ is a (Reichenbachian) common cause of the correlation (1) if it satisfies the following conditions

$$
\begin{aligned}
\mu(A \cap B \mid C) & =\mu(A \mid C) \mu(B \mid C) \\
\mu\left(A \cap B \mid C^{c}\right) & =\mu\left(A \mid C^{c}\right) \mu\left(B \mid C^{c}\right) \\
\mu(A \cap C) & >\mu\left(A \cap C^{c}\right) \\
\mu(B \cap C) & >\mu\left(B \cap C^{c}\right)
\end{aligned}
$$

Reichenbach also formulated what is called the

Common Cause Principle: If two events $A, B$ are correlated then either there is a direct causal link between $A$ and $B$ that is responsible for the correlation, or, if $A$ and $B$ are causally independent, then there exists a common cause that explains the correlation.

Viewed from the perspective of the Common Cause Principle, the definition of common cause (Definition 2) is incomplete for two reasons:

(i) The definition does not contain an explicit requirement about the causal independence of $A$ and $B$.

(ii) It is part of the intuition about the Common Cause Principle that the common cause $C$ should be an event that can causally affect events $A$ and $B$; however, there is no explicit expression of this type of nonprobabilistic causal link between $C$ and $A$ and $C$ and $B$ in the definition of common cause. 
How should one transform the two informal conditions (i) and (ii) above into precise and technically explicit conditions in order to obtain a formulation of the Common Cause Principle that can be formally analyzed? In our view this is the deepest and most important problem in connection with the Common Cause Principle, which lies at the heart of most of the debates about the Common Cause Principle. It is here that Henson's framework and the notion of least domain of decidability offers a solution. Before recalling how, we need to make two remarks about possible modifications of the notion of common cause:

The first remark is that there are arguments in favor of weakening the notion of common cause in the following sense: It is conceivable that a correlation is due not to a single common cause but a whole set of "partial common causes". This idea leads to the notion of common cause system, which was analyzed in a number of publications (see [Uff99], HSR04, HSR06], [WM10]). A common cause system is a partition $\left\{C_{i}: i \in I\right\}$ of $\Omega$ such that

$$
\begin{gathered}
\mu\left(A \cap B \mid C_{i}\right)=\mu\left(A \mid C_{i}\right) p\left(B \mid C_{i}\right) \quad \text { for all } \quad i \in I \\
{\left[\mu\left(A \mid C_{i}\right)-\mu\left(A \mid C_{j}\right)\right]\left[\mu\left(B \mid C_{i}\right)-\mu\left(B \mid C_{j}\right)\right]>0 \quad(i \neq j)}
\end{gathered}
$$

Equation (6) is called the screening-off condition, (7) is the analogue of the statistical relevance conditions (44)-(5) (see [HSR04, [HSR06] for a further motivation of condition (77)).

The second remark is that when it comes to proving that common causes cannot exist for certain correlations, one would like to prove such "no-go" theorems under the weakest conditions; hence, frequently the general statistical relevance condition (77) is dropped from the definition of common cause system - this is what Henson also does in the formulation of the two causality principles SO1 and SO2. These principles are as follows.

SO1. For all events $A$ and $B$ with $\operatorname{dom}(A) \subset \mathscr{A}$ and $\operatorname{dom}(B) \subset \mathscr{B}$, if $\mathscr{A}$ and $\mathscr{B}$ are space-like separated regions, then

$$
\mu(A \cap B \mid C)=\mu(A \mid C) \mu(B \mid C) \quad \forall C \in \Phi\left(\mathscr{P}_{1}(\mathscr{A}, \mathscr{B})\right) .
$$

SO2. For all events $A$ and $B$ with $\operatorname{dom}(A) \subset \mathscr{A}$ and $\operatorname{dom}(B) \subset \mathscr{B}$, if $\mathscr{A}$ and $\mathscr{B}$ are space-like separated regions then:

$$
\mu(A \cap B \mid C)=\mu(A \mid C) \mu(B \mid C) \quad \forall C \in \Phi\left(\mathscr{P}_{2}(\mathscr{A}, \mathscr{B})\right) .
$$

Some remarks on SO1 and SO2 are in order:

\section{Remark 1.}

(i) Note that the $C$ 's referred to in $\mathrm{SO} 1$ and $\mathrm{SO} 2$ do form a partition because they are full specifications of regions and these form a partition by Proposition 1; hence both SO1 and SO2 state that correlations between events with causally disjoint domains of decidability have a common cause system. 
(ii) The fact that $C$ 's are required to be full specifications in the respective regions entails (and thus is motivated by) the fact that this way "Simpson events", events that could re-introduce the correlation between $A$ and $B$ after they have been screened off by $C$ 's, are excluded (see [Hen05, p. 526-527] for a further discussion of the "Simpson problem").

(iii) The two principles differ in which past they require the postulated screening-off partition to be located: The first principle requires the common cause system to lie in the mutual past of $A$ and $B$ : recall that $\mathscr{P}_{1}(\mathscr{A}, \mathscr{B})=J^{-}(\mathscr{A}) \cap J^{-}(\mathscr{B})$. The second principle requires the common cause system to be localized in the truncated joint past of $A$ and $B: \mathscr{P}_{2}(\mathscr{A}, \mathscr{B})=\left(J^{-}(\mathscr{A}) \cup J^{-}(\mathscr{B})\right) \backslash(\mathscr{A} \cup \mathscr{B})$.

(iv) There is a deceptive dissimilarity in requiring the common cause system in SO1 to be in the untruncated mutual past and to be in the truncated joint past in $\mathrm{SO} 2$; however, since $\mathscr{A}$ and $\mathscr{B}$ are assumed to space-like separated the untruncated and truncated mutual pasts of $\mathscr{A}$ and $\mathscr{B}$ coincide; hence there is no need to require explicitly the common cause system to be in the truncated mutual past. But one has to do so explicitly in SO2 because, intuitively, we do not want the common cause system to be in the regions where the correlated events are. Requiring the common cause system to be in the truncated mutual past excludes for instance the common cause system to be the partition generated by $A$ and $B$.

(v) The difference between the two localizability regions in SO1 and SO2 is that

- The mutual past is the set of points in $\mathbb{S}$ each of which is causally related to at least one point in both $\mathscr{A}$ and $\mathscr{B}$.

- The truncated joint past consist of the set of points in $\mathbb{S}$ none of which is in either $\mathscr{A}$ or in $\mathscr{B}$, and each of which is causally related to at least one point in either $\mathscr{A}$ or $\mathscr{B}$.

The description (v) above of the mutual and joint pasts indicates that SO1 and SO2 are different: SO2 seems a weaker principle than SO1. Henson claims however that SO1 and SO2 are equivalent (Corollary 2 in Hen05]). We now sketch the proof of this equivalence claim. In the proof the following two regions $\mathscr{X}$ and $\mathscr{Y}$ will be referred to:

$$
\begin{aligned}
\mathscr{X} & =\left[J^{-}(\mathscr{A}) \backslash \mathscr{A}\right] \backslash J^{-}(\mathscr{B}) \\
\mathscr{Y} & =\left[J^{-}(\mathscr{B}) \backslash \mathscr{B}\right] \backslash J^{-}(\mathscr{A})
\end{aligned}
$$

(I). Proof of $\mathrm{SO} 1 \Rightarrow \mathrm{SO} 2$

Assume SO1. Then for $A$ and $B$ such that $\operatorname{dom}(A) \subset \mathscr{A}, \operatorname{dom}(B) \subset \mathscr{B}$ 
and $\mathscr{A}$ and $\mathscr{B}$ space-like separated we have:

$$
\mu(A \cap B \mid C)=\mu(A \mid C) \mu(B \mid C) \quad \forall C \in \Phi\left(\mathscr{P}_{1}(\mathscr{A}, \mathscr{B})\right),
$$

Let $X$ and $Y$ be full specifications of regions $\mathscr{X}$ and $\mathscr{Y}$, respectively, and consider the following four pairs of events

$$
\{(A \cap X),(B \cap Y)\} \quad\{(A \cap X), B\} \quad\{A,(B \cap Y)\} \quad\{X, Y\}
$$

It is easy to show that the two events in each of the above pairs occur in regions $\{(\mathscr{A} \cup \mathscr{X}),(\mathscr{B} \cup \mathscr{Y})\}$, which are space-like separated; on the other hand the mutual past of $(\mathscr{A} \cup \mathscr{X})$ and $(\mathscr{B} \cup \mathscr{Y})$ is equal to the mutual past of $\mathscr{A}$ and $\mathscr{B}$ :

$$
J^{-}(\mathscr{A} \cup \mathscr{X}) \cap J^{-}(\mathscr{B} \cup \mathscr{Y})=J^{-}(\mathscr{A}) \cap J^{-}(\mathscr{B})
$$

Thus SO1 can be applied to each pair in eq. (13) and we obtain that any full specification $C$ of $\Phi\left(\mathscr{P}_{1}(\mathscr{A}, \mathscr{B})\right)$ screens off the correlation between the elements in every of these pairs.

A probability Lemma [Hen05, Lemma 4, p. 528] is then invoked to show that $\forall X \in \Phi(\mathscr{X}), \forall Y \in \Phi(\mathscr{Y})$ and $C \in \Phi\left(\mathscr{P}_{1}(\mathscr{A}, \mathscr{B})\right)$ the following relation follows:

$$
\mu(A \mid X \cap Y \cap C) \mu(B \mid X \cap Y \cap C)=\mu(A \cap B \mid X \cap Y \cap C) .
$$

On the other hand, a full specification $F$ of a region $\mathscr{R}$ defined as the disjoint union of some finite set of regions $\mathscr{A}_{i}$, i.e. $\mathscr{R}=\sqcup_{i} \mathscr{A}_{i}$, can be written as $F=\cap_{i} A_{i}$, where $A_{i}$ is a full specification of $\mathscr{A}_{i}$ [Hen05, Corollary 1, p. 525].

Then, since $X, Y$ and $C$ are full specifications of $\mathscr{X}, \mathscr{Y}$ and $\Phi\left(\mathscr{P}_{1}(\mathscr{A}, \mathscr{B})\right)$ respectively, i.e. $X \in \Phi(\mathscr{X}), Y \in \Phi(\mathscr{Y})$ and $C \in \Phi\left(\mathscr{P}_{1}(\mathscr{A}, \mathscr{B})\right)$, and since $\mathscr{P}_{2}(\mathscr{A}, \mathscr{B})=\mathscr{X} \sqcup \mathscr{Y} \sqcup \mathscr{P}_{1}$, we have that

$$
C \cap X \cap Y \in \Phi\left(\mathscr{P}_{2}(\mathscr{A}, \mathscr{B})\right) .
$$

It is concluded then that SO1 implies SO2.

(II). $\mathrm{SO} 2 \Rightarrow \mathrm{SO} 1$

Assume now SO2. Thus if $A$ and $B$ are such that $\operatorname{dom}(A) \subset \mathscr{A}$, $\operatorname{dom}(B) \subset \mathscr{B}$ and $\mathscr{A}$ and $\mathscr{B}$ are spacelike separated. Then we have:

$$
\mu(A \cap B \mid C)=\mu(A \mid C) \mu(B \mid C) \quad \forall C \in \Phi\left(\mathscr{P}_{2}(\mathscr{A}, \mathscr{B})\right)
$$

Consider again the regions $\mathscr{X}$ and $\mathscr{Y}$ defined by eqs. (10)-(11). 
Now if $\operatorname{dom}(A) \subset \mathscr{A}$ then we also have that $\operatorname{dom}(A) \subset(\mathscr{A} \cup \mathscr{X})$. Similarly if $\operatorname{dom}(B) \subset \mathscr{B}$ then $\operatorname{dom}(B) \subset(\mathscr{B} \cup \mathscr{Y})$. This means that events $A$ and $B$ occur in regions $\{(\mathscr{A} \cup \mathscr{X}),(\mathscr{B} \cup \mathscr{Y})\}$ which, as before, can be seen to be space-like separated. Thus we can apply SO2 to these two regions. If we calculate the truncated joint past of the regions $(\mathscr{A} \cup \mathscr{X})$ and $(\mathscr{B} \cup \mathscr{Y})$ we find however that it coincides with the mutual past of the regions $\mathscr{A}$ and $\mathscr{B}$, i.e.

$$
\mathscr{P}_{2}((\mathscr{A} \cup \mathscr{X}),(\mathscr{B} \cup \mathscr{Y}))=\mathscr{P}_{1}(\mathscr{A}, \mathscr{B})
$$

This means that eq. (9) is the same as eq. (8); hence $\mathrm{SO} 2 \Rightarrow \mathrm{SO} 1$.

\section{$4 \quad$ Finite and infinite $\mathrm{SO} 1$ and $\mathrm{SO} 2$}

The equivalence of SO1 and SO2 does not seem to fit well with intuition: Existence of an explanation of a correlation in terms of a common cause system that is localized in the mutual past of the correlated events does not seem to be a priori inferable from an explanation in terms of common causes of which we only know that they are localized in the (truncated) joint past, which is typically a larger region; yet, the equivalence, if holds, entails that we are licensed to make such an inference. How can one reconcile the equivalence claim with this intuition?

The proof of the equivalence claim shows that the following two conditions are crucial in showing the equivalence:

Loose domain: The screening-off conditions (8)-(9) in SO1 and SO2 are required not for the full specifications of the intersection and truncated joint past of the least domains $\operatorname{dom}(A)$ and $\operatorname{dom}(B)$ of the correlated events $A$ and $B$ but for the full specifications of the intersection and truncated past of all spacelike separated regions $\mathscr{A}$ and $\mathscr{B}$ containing the least domains $\operatorname{dom}(A)$ and $\operatorname{dom}(B)$, respectively.

Tricky regions: Because of the above Loose domain property of the principles $\mathrm{SO} 1$ and $\mathrm{SO} 2$, in the proof $\mathrm{SO} 2 \Rightarrow \mathrm{SO} 1$ it is allowed that $\mathrm{SO} 2$ is applied to regions of the form $(\mathscr{A} \cup \mathscr{X})$ and $(\mathscr{B} \cup \mathscr{Y})$ that have the peculiar property that their truncated joint past is equal to the mutual past of $\mathscr{A}$ and $\mathscr{B}$. This is achieved by taking $\mathscr{X}$ and $\mathscr{Y}$ as defined by eqs. (10)-(11).

Henson himself notes [Hen05, p. 527] that the Loose domain property is somewhat unnatural and needs justification because the natural condition would be to require the screening-off conditions (8)-(9) to hold for the full specifications of the intersection and truncated joint past of the least domains of decidability $\operatorname{dom}(A)$ and $\operatorname{dom}(B)$ of the correlated events $A$ and $B$, not for the full specifications of all regions $\mathscr{A}$ and $\mathscr{B}$ containing the least 


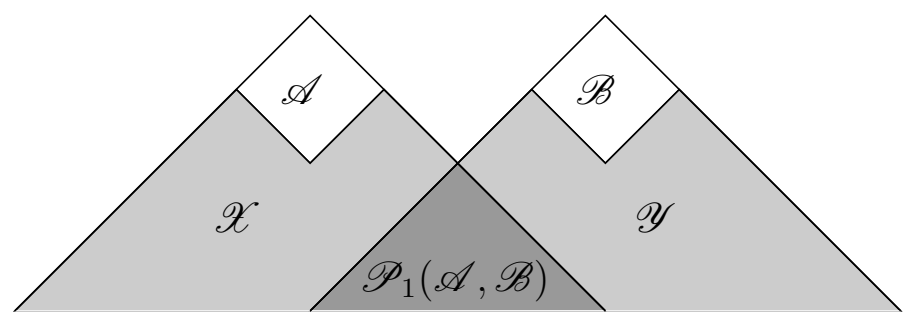

Figure 1: The truncated joint past of $(\mathscr{A} \cup \mathscr{X})$ and $(\mathscr{B} \cup \mathscr{Y})$ is equal to the mutual past of $\mathscr{A}$ and $\mathscr{B}$

domains of decidability. The argument Henson provides for the justification is that if one requires the screening-off conditions for disjoint events $A_{i}$ all having least domain of decidability to be equal to $\mathscr{A}$ then this entails (by the properties of the least domain of decidability function dom) that the screening-off conditions hold for events of the form $A=\cup A_{i}$ and these events have their least domain of decidability just contained but not necessarily equal to $\mathscr{A}$. Henson points out that such a justification "... can always be constructed for any $\mathscr{A} \supset \operatorname{dom}(A)$ as long as there are events with $\mathscr{A}$ as their least domain of decidability" [Hen05, p. 527] (our emphasis).

In particular such a justification is needed in case of the tricky domains $(\mathscr{A} \cup \mathscr{X})$ and $(\mathscr{B} \cup \mathscr{Y})$ that feature in the proof of the equivalence claims because these regions are strictly larger than the least domains of decidability $\mathscr{A}$ and $\mathscr{B}$. Note however that the regions $(\mathscr{A} \cup \mathscr{X})$ and $(\mathscr{B} \cup \mathscr{Y})$ are in a sense causally very large. For a typical example, take $\mathbb{S}$ to be the Minkowski spacetime $M$ : Let $\mathscr{A}$ and $\mathscr{B}$ be two spacelike separated double cones. Then $J^{-}(\mathscr{A}) \backslash \mathscr{A}$ is the infinitely large region of the full causal past of the double cone $\mathscr{A}$ minus the finite double cone subtracted from it, and $\left(J^{-}(\mathscr{A}) \backslash \mathscr{A}\right) \backslash J^{-}(\mathscr{B})$ is the also infinitely large strip that remains of $J^{-}(\mathscr{A}) \backslash \mathscr{A}$ after subtracting from it the full past of $\mathscr{B}$. It is then clear why the truncated joint past of $(\mathscr{A} \cup \mathscr{X})$ and $(\mathscr{B} \cup \mathscr{Y})$ is equal to the mutual past of the double cones $\mathscr{A}$ and $\mathscr{B}$ (see Fig. (1).

Adjectives "finite" and "infinite" in the previous sentences are used in the sense of a metric, which is available in Minkowski spacetime: a double cone $\mathscr{A}$ is finite in the sense of the metric, $\left(J^{-}(\mathscr{A}) \backslash \mathscr{A}\right) \backslash J^{-}(\mathscr{B})$ is not. But a metric is not assumed to be part of a general causal set, so finiteness and infiniteness cannot be defined in metric terms. On the basis of the example of double cones in Minkowski spacetime one can however formulate, in terms of the causal order only, what is important: subtracting a double cone from its causal past is nonempty; in addition, a double cone is causally closed in the sense of being equal to its causal closure, where causal closure of a spacetime region $O$ is, by definition, the set $\left(O^{\prime}\right)^{\prime}$ where $O^{\prime}$, the causal complement of $O$, is the set of points in the Minkowski spacetime that are spacelike from every 
Are the four causality principles equivalent?

point in $O$. Furthermore, every bounded region $\mathscr{A}$ in Minkowski spacetime is contained in a double cone, the smallest of such double cones is equal to the causal closure of $\mathscr{A}$. The notion of causal complement and causal closure makes perfect sense in any causal set $\mathbb{S}$ in place of the Minkowski spacetime, and all this motivates the following definition.

Definition 3. A region $\mathscr{A}$ in $\mathbb{S}$ is called causally finite if the causal past of its causal closure (denoted by $\overline{\mathscr{A}}$ ) is strictly larger than itself, i.e. if it holds that

$$
J^{-}(\overline{\mathscr{A}}) \backslash \overline{\mathscr{A}} \neq \emptyset
$$

The region is called causally infinite if it is not causally finite.

As the case of Minkowski spacetime indicates the regions $(\mathscr{A} \cup \mathscr{X})$ and $(\mathscr{B} \cup \mathscr{Y})$ can be causally infinite in the sense of the above definition and since SO1 and SO2 are applied to them, Henson's justification why this is conceptually admissible needs to be invoked, which, as we saw, assumes that there are events with $(\mathscr{A} \cup \mathscr{X})$ and $(\mathscr{B} \cup \mathscr{Y})$ as least domains of decidability. Causally finite and causally infinite regions are conceptually very different however if considered as least domains of decidability: an event whose least domain of decidability is equal to a causally infinite set $\mathscr{Z}$ is only comprehendible by taking into account the complete causal structure of the region $\mathscr{Z}$. This whole region might very well be empirically inaccessible in its entirety however (think of the case of the double cones in Minkowski spacetime); hence one might not be in the position to decide empirically whether such an event has happened or not. In particular, observing a correlation involving such an event might very well be problematic.

Thus it is conceptually well-motivated to distinguish two versions of both SO1 and SO2: the original ones, in which no restrictions are imposed on the regions, call these principles infinite $\mathrm{SO} 1$ and infinite $\mathrm{SO} 2$, and ones in which $\mathscr{A}$ and $\mathscr{B}$ are restricted to causally finite regions:

Finite SO1. For all events $A$ and $B$ with $\operatorname{dom}(A) \subset \mathscr{A}$ and $\operatorname{dom}(B) \subset \mathscr{B}$, if $\mathscr{A}$ and $\mathscr{B}$ are causally finite space-like separated regions, then

$$
\mu(A \cap B \mid C)=\mu(A \mid C) \mu(B \mid C) \quad \forall C \in \Phi\left(\mathscr{P}_{1}(\mathscr{A}, \mathscr{B})\right) .
$$

Finite SO2. For all events $A$ and $B$ with $\operatorname{dom}(A) \subset \mathscr{A}$ and $\operatorname{dom}(B) \subset \mathscr{B}$, if $\mathscr{A}$ and $\mathscr{B}$ are causally finite space-like separated regions then:

$$
\mu(A \cap B \mid C)=\mu(A \mid C) \mu(B \mid C) \quad \forall C \in \Phi\left(\mathscr{P}_{2}(\mathscr{A}, \mathscr{B})\right) .
$$

\section{Are the four causality principles equivalent?}

What is the relation of the four causality principles finite and infinite SO1 and finite and infinite $\mathrm{SO} 2$ ? 


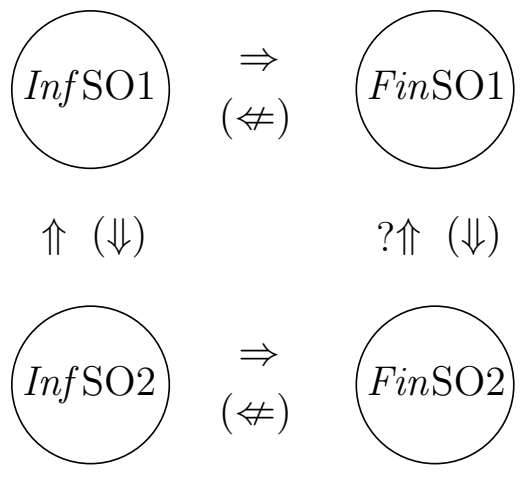

Figure 2: Logical relations between the diverse screening-off principles. Relations in parenthesis are considered as likely to hold, although no complete formal proof has been found. A question mark indicates that the corresponding logical relation is not known.

As we recalled in Section 3. Henson has shown that infinite SO2 entails infinite SO1. As the example of double cones in Minkowski space shows, the region $(\mathscr{A} \cup \mathscr{X})$, where $\mathscr{X}$ is defined by eq. (10), may not be causally finite even if both $\mathscr{A}$ and $\mathscr{B}$ are. Consequently, the proof presented in Section 3 of the implication infinite $\mathrm{SO} 2 \Rightarrow$ infinite $\mathrm{SO} 1$, which relies in a crucial way on applying SO2 to regions of the form $(\mathscr{A} \cup \mathscr{X})$, does not prove the implication finite $\mathrm{SO} 2 \Rightarrow$ infinite $\mathrm{SO} 1$, not even the implication finite $\mathrm{SO} 2 \Rightarrow$ finite $\mathrm{SO} 1$.

What Henson's argument in Section 3 in favor of the implication SO1 $\Rightarrow \mathrm{SO} 2$ shows, strictly speaking, is that, given any pair $A, B$, and $C \in$ $\Phi\left(\mathscr{P}_{1}(\mathscr{A}, \mathscr{B})\right)$ satisfying (8)), there is always a $C^{\prime} \in \mathscr{P}_{2}(\mathscr{A}, \mathscr{B})$ (namely $\left.C^{\prime}=C \cap X \cap Y\right)$ such that $A, B$ and $C^{\prime}$ satisfy the condition required by $\mathrm{SO} 2$. This does not yield yet that SO1 entails SO2, however: in order to infer this, one needs to show that these $C^{\prime} \in \mathscr{P}_{2}(\mathscr{A}, \mathscr{B})$ events exhaust the set $\Phi\left(\mathscr{P}_{2}(\mathscr{A}, \mathscr{B})\right)$ of all possible full specifications of $\mathscr{P}_{2}(\mathscr{A}, \mathscr{B})-$ and it remains unclear why this is the case, especially in case of causally infinite least domains of decidability. Thus we regard the problem of whether the implication [(finite or infinite) $\mathrm{SO} 1 \Rightarrow$ (finite or infinite) SO2] as open but likely true and intuitively plausible.

The implications [infinite $\mathrm{SO} 1 \Rightarrow$ finite $\mathrm{SO} 1$ ] and [infinite $\mathrm{SO} 2 \Rightarrow$ finite $\mathrm{SO} 2]$ are trivial. The diagram in Fig. 2 summarizes the logical relations known between these causality principles at this time, question marks indicating open problems.

The truth of the claim formulated above that Henson's proof in Section 3 of the implication [infinite $\mathrm{SO} 2 \Rightarrow$ infinite $\mathrm{SO} 1$ ] does not prove either of the implications [finite $\mathrm{SO} 2 \Rightarrow$ infinite $\mathrm{SO} 1$ ] or [finite $\mathrm{SO} 2 \Rightarrow$ finite $\mathrm{SO} 1$ ] does not entail that none of these implications is true, and we do not have a 
Are the four causality principles equivalent?

formal proof that these implications do not hold. But the analysis in local algebraic relativistic quantum field theory (AQFT) of the status of SO1 and $\mathrm{SO} 2$ yields a strong argument that finite $\mathrm{SO} 2$ is strictly weaker than infinite $\mathrm{SO} 2$. So we conclude by commenting on the status of the causality principles $\mathrm{SO} 1$ and SO2 in local algebraic relativistic quantum field theory (AQFT).

It was shown in RS02, RS07, (see also Réd97, chapter in Réd98 and Réd02]) that if AQFT satisfies the condition of local primitive causality, then finite SO2 holds in AQFT in the specific sense that there is a single common cause of spacelike correlations predicted by faithful states between projections contained in algebras pertaining to spacelike separated double cones and the common cause is localized in the truncated joint past of the backward light cones of the double cones containing the correlated projections (Proposition 3 in [RS02]). This fact was referred to by saying that AQFT satisfies the weak common cause principle. It could not be proved so far however that AQFT also satisfies (even the finite) SO1. In view of the distinctions made here between finite and infinite versions of SO1 and $\mathrm{SO} 2$, it is instructive to see why the proof in $\mathrm{RS} 02$ of the claim that AQFT satisfies the weak common cause principle is too weak to prove that infinite $\mathrm{SO} 2$ holds in AQFT, because the proof shows how radically different finite and infinite versions of $\mathrm{SO} 2$ and $\mathrm{SO} 1$ can be.

The proof of finite $\mathrm{SO} 2$ in AQFT consists of the following steps:

step 1 One shows, utilizing the special type of the local algebras pertaining to double cones that the probability theories describing the observables represented by these algebras have the particular measure theoretic feature of being atomless.

step 2 One proves that atomlessness entails that there exists a projection in the smallest double cone $\mathscr{C}$ containing the double cones $\mathscr{A}$ and $\mathscr{B}$ which satisfies the probabilistic conditions required of a common cause.

step 3 Using the local primitive causality condition one "pushes back" the common cause in $\mathscr{C}$ far enough back in the joint causal past of $\mathscr{A}$ and $\mathscr{B}$ until it is in the truncated joint past of $\mathscr{A}$ and $\mathscr{B}$.

(Recall that the local primitive causality condition states that the local algebra $\mathcal{A}(V)$ of observables associated with an open bounded spacetime region $V$ is equal to the local algebra of observables $\mathcal{A}(\bar{V})$ where $\bar{V}$ is the causal closure of $V$.)

This proof cannot be extended to conclude that infinite $\mathrm{SO} 2$ also holds in AQFT, for the following reasons:

- While it could be proved that local algebras associated to double cone regions have the measure theoretic atomlessness as a consequence of 
such algebras being type III von Neumann algebras, this latter algebraic feature is only known to hold in AQFT for algebras pertaining to special spacetime regions and hence cannot be assumed for arbitrary regions such as (10)-(11). That is to say, already step 1 is not known to be valid for arbitrary, in particular causally infinite, regions.

Moral: the type of measure space describing events may very well restrict the possible shapes of regions that are least domains of decidability for the events in the probability space.

- Step 3 only works because double cones are causally finite (in the sense of Definition [3): Even if we could make step 1 for regions $\mathscr{A} \cup \mathscr{X}$ and $\mathscr{B} \cup \mathscr{Y}$ and did find a common cause in the untruncated joint past of $\mathscr{A} \cup \mathscr{X}$ and $\mathscr{B} \cup \mathscr{Y}$ ( $\mathscr{X}$ and $\mathscr{Y}$ defined by (10) and (11)), we would not be able to "push back" this common cause far enough in the untruncated joint past of $\mathscr{A} \cup \mathscr{X}$ so that it gets in the truncated joint past of $\mathscr{A} \cup \mathscr{X}$ and $\mathscr{B} \cup \mathscr{Y}$ - precisely because these regions are causally infinite and thus there is no open bounded (hence causally finite) region $V$ having the property that its causal closure $\bar{V}$ is equal to the untruncated joint past of $\mathscr{A} \cup \mathscr{X}$ and $\mathscr{B} \cup \mathscr{Y}$.

Thus the situation in AQFT indicates that finite and infinite $\mathrm{SO} 2$ are not equivalent and thus that the logical relationships among these different causality principles is a very subtle and complicated matter, clarification of their relation requiring further work.

\section{Acknowledgements}

Research supported by the Hungarian Scientific Research Fund (OTKA), contract number: K100715; and by the Spanish Ministry of Science and Innovation (MICINN) Research Project FFI2008-06418-C01-03.

\section{References}

[Hen05] J. Henson, Comparing causality principles, Studies in the History and Philosophy of Modern Physics 36 (2005), 519-543.

[HSR04] G. Hofer-Szabó and M. Rédei, Reichenbachian common cause systems, International Journal of Theoretical Physics 43 (2004), $1819-1826$.

[HSR06] — Reichenbachian common cause systems of arbitrary finite size exist, Foundations of Physics Letters 35 (2006), 745-746.

[HSV12] G. Hofer-Szabó and P. Vecsernyés, Reichenbach's common cause principle in algebraic quantum field theory with locally finite degrees of freedom, Foundations of Physics 42 (2012), 241-255. 
[Réd97] M. Rédei, Reichenbach's Common Cause Principle and quantum field theory, Foundations of Physics 27 (1997), 1309-1321.

[Réd98] _ Q Quantum logic in algebraic approach, Fundamental Theories of Physics, vol. 91, Kluwer Academic Publisher, 1998.

[Réd02] _ Reichenbach's common cause principle and quantum correlations, Modality, Probability and Bell's Theorems (T. Placek and J. Butterfield, eds.), NATO Science Series, II., vol. 64, Kluwer Academic Publishers, Dordrecht, Boston, London, 2002, pp. 259270 .

[Rei56] H. Reichenbach, The direction of time, University of California Press, Los Angeles, 1956.

[RS02] M. Rédei and S.J. Summers, Local primitive causality and the common cause principle in quantum field theory, Foundations of Physics 32 (2002), 335-355.

[RS07] , Remarks on causality in relativistic quantum field theory, International Journal of Theoretical Physics 46 (2007), 2053-2062.

[Sor03] R.D. Sorkin, Causal sets: Discrete gravity (Notes for the Valdivia Summer School), 2003, http://arxiv.org/abs/gr-qc/0309009.

[Uff99] J. Uffink, The principle of the common cause faces the Bernstein paradox, Philosophy of Science, Supplement 66 (1999), 512-525.

[WM10] L. Wronski and M. Marczyk, Only countable Reichenbachian common cause systems exist, Foundations of Physics 40 (2010), 11551160. 